\title{
L'allergie aux alliages dentaires non précieux : \\ données de la littérature et solutions actuelles
}

\section{Allergy in the non-precious dental alloys: data of the literature and current solutions}

\section{MOTS-CLEFS :}

- Allergie et immunologie, Hypersensibilité, Manifestations orales, Alliages dentaires, Nickel

\section{KEYWORDS:}

- Allergy and Immunology, Hypersensitivity, Oral manifestations, Dental alloys, Nickel

$\operatorname{AOS} n^{\circ} 275-2016$

\section{Résumé}

Les alliages dentaires non précieux peuvent être ò l'origine de sensibilisations et allergies. Les alliages base nickel ont un plus fort potentiel de sensibilisation que les alliages base cobalt. Les réactions d'hypersensibilité au nickel apparaitraient après une sensibilisation primaire par un contact non dentaire. Il est aussi remarqué que la présence intra-orale d'un allergène connu pourrait améliorer ou induire une tolérance immunologique envers cet allergène. Nous présentons ici les manifestations orales des réactions allergiques, rares et de diagnostic difficile mais aussi manifestations ò distance. Quelques conseils de prise en charge odontologique des patients sont donnés avec les directives européennes en vigueur. La demande croissante de matériaux plus biocompatibles a conduit à l'introduction de nouveaux aciers inoxydables sans ou avec un faible contenu en nickel. Dans les pays européens où les alliages $\mathrm{NiCr}$ (nickel $\leq 1 \%$ poids) sont encore autorisés, ils sont moins utilisés au profit des alliages $\mathrm{CoCr}$.
Abstract

Non-precious dental alloys can be at the origin of sensitizations and allergies. Nickel base alloys present higher potential of sansitization than cobalt base alloys. Nickel hypersensitivity's reactions would happen after a primary sensitization by a non dental contact. It is also noticed that intra-oral presence of a known allergen could improve or induce an immunological tolerance to this allergen. We present here the oral demonstrations of the allergical reactions, rare with difficult diagnosis but also remote demonstrations. Some advices of odontological care of the patients are given with the current European directives. The increasing request of more biocompatible materials led to the introduction of news stainless steels with less or without nickel content. In european countries where $\mathrm{NiCr}$ alloys (nickel $\leq 1 \%$ weight) are yet authorized, they are less employed to the benefit of CoCr alloys.

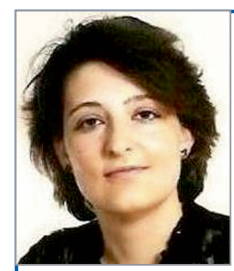

- Sylvie BEAUFILS, docteur en chirurgie dentaire, chargée d'enseignement, ancienne AHU, DESCB, Master Sciences, technologie, santé, sciences pour l'ingénieur, D.U. dermato-vénéréologie de la muqueuse buccale, UFR Odontologie, 51100 Reims, beaufils.sylvie@etudiant.univ-reims.fr.

Pauline PIERRON, docteur en chirurgie dentaire, Mutualité Française ChampagneArdenne, o8300 Rethel.

Pierre MILLET, docteur en chirurgie dentaire, PU-PH, Pôle Odontologie- $\mathrm{CHU}$, 51100 Reims, UFR Odontologie, 51100 Reims. 


\section{INTRODUCTION}

Depuis les années 1965-70 dans le monde, les alliages non précieux se sont majoritairement et progressivement substitués aux alliages précieux et semi-précieux dans les prothèses fixes et amovibles pour des raisons économiques et de par leurs propriétés mécaniques excellentes. Toutefois, ils présentent une résistance à la corrosion plus faible et des problèmes de biocompatibilité. L'allergie est une cause reconnue de réactions cliniques, notamment réponses inflammatoires, face à des alliages dentaires coulés. Les prothèses, les implants, les dispositifs orthodontiques faits d'alliages non précieux peuvent être à l'origine de sensibilisation et allergies. Non allergènes par eux-mêmes, ils relarguent des allergènes (métaux et leurs sels) lors de leur dégradation mécanique, chimique ou électrochimique (corrosion). Patients, chirurgiens-dentistes et prothésistes dentaires sont concernés. Nous présentons ici les manifestations orales et à distance des réactions allergiques et l'influence de la nature du métal. Quelques conseils de dépistage et prise en charge odontologique des patients sont donnés avec les directives européennes en vigueur. Des solutions à l'allergie avec de nouveaux matériaux moins allergènes, moins corrodables sont présentées aussi.

\section{CLASSIFICATION, INDICATIONS ET COMPOSITIONS DES ALLIAGES DENTAIRES NON PRÉCIEUX}

Les alliages dentaires « base métal » ou alliages non précieux comprennent moins de $25 \%$ en poids de métaux nobles. Il n'existe aucune classification officielle. En France, la classification utilisée reprend celle des normes internationales (NF EN ISO 1562, 8891 et 9693). On peut distinguer 5 grandes familles :

\section{Alliages à base nickel $(\mathrm{NiCr}, \mathrm{NiCrBe}$, NiCrMo) ou « superalliages »}

Interdits dans certains pays européens, ils sont remis en cause par leur toxicité, le potentiel allergisant du nickel avec des inquiétudes sur leur biocompatibilité.

\section{Alliages à base cobalt $(\mathrm{CoCr}, \mathrm{CoCrMo}$, CoCrW, CoCrNiMo) ou « stellite »}

Ils présentent une meilleure résistance à la corrosion, une moindre allergénicité et une meilleure biocompatibilité. Ils se substituent actuellement aux alliages $\mathrm{Ni}-\mathrm{Cr}$ et sont les alliages les plus utilisés en Europe et au Japon.
Alliages à base fer

(NiCrFe ; principalement aciers inoxydables austénitiques)

Ils sont maintenant très employés en dentisterie et présentent une excellente biocompatibilité et une forte résistance à la corrosion.

\section{Alliages à base cuivre (CuSn, CuZn, CuAl, CuAINi)}

Rarement employés, plus économiques mais aussi plus toxiques et corrodables, ils sont ou ont été utilisés en Europe de l'Est, USA, Amérique latine et Japon.

\section{Alliages de titane ( $\left.\mathrm{TA}_{6} \mathrm{~V}, \mathrm{TMA}, \mathrm{NiTi}\right)$ et titane pur}

Ils présentent généralement une excellente biocompatibilité et une faible corrosion.

Les alliages non précieux sont indiqués selon leur nature et leurs propriétés pour diverses applications odontologiques: reconstitutions corono-radiculaires ou "inlay core ", prothèses fixes plurales et unitaires (couronnes, bridges, infrastructure des restaurations céramométalliques, facettes, "inlay", " onlay ", " overdentures ", tenons façonnés), châssis et infrastructures de prothèses amovibles (overlays, crochets coulés...), dispositifs orthodontiques (fils, ligatures, arcs, bagues, brackets, micro-vis), dispositifs implantaires (implants, barres implanto-portées, structures supra-implantaires, attachements, systèmes de rétention magnétique), prothèses articulaires (ATM) et dans l'instrumentation dentaire (instruments chirurgicaux, instruments rotatifs (contreangle, turbine...), fraises, limes endodontiques, forêts). Les alliages à base nickel sont privilégiés pour l'élaboration des « inlay-core » et des prothèses fixes. Les alliages base cobalt sont généralement destinés à la confection de châssis ou infrastructures de prothèses amovibles, « overlays ", crochets coulés, « overdentures » et implants. Dans les bridges de longues portées et faible hauteur coronaire ou pour les prothèses composites à attachement, ces alliages sont maintenant préférés. De nouveaux alliages base cobalt ont été développés pour la technique céramo-métallique. La tête du composant condylaire des prothèses articulaires totales est généralement faite d'alliages CoCr. Des tenons façonnés sont réalisés en acier inoxydable, alliages $\mathrm{Cu}-\mathrm{Zn}$ ou Co-Cr-Ni. Les alliages $\mathrm{CuAl}$ (Duracast ${ }^{\circledR}$...) sont devenus populaires pour les tenons et reconstitutions coronaires coulées. Les aciers inoxydables (principalement aciers austénitiques) sont privilégiés pour les dispositifs orthodontiques (brackets, bagues, arcs orthodontiques, micro-implants, micro-vis, fils tressés ou torsadés, fils " Tru-chrome» et fils australiens) et instruments 
endodontiques, mais utilisés aussi dans les assemblages, attachements, systèmes de rétention magnétiques, overdentures implanto-portées, couronnes préformées, couronnes et bridges. Les éléments orthodontiques fixes : brackets, ressorts, attelles, bagues, ligatures et arcs orthodontiques, utilisent majoritairement des alliages coulés ou façonnés à base de fer ou de cobalt, des alliages NiCo. Les fils Elgiloy en alliages CoCr sont appréciés pour les arcs à boucles multiples. L'alliage $\mathrm{Sm}_{2} \mathrm{Co}_{17}$ est utilisé dans les aimants à vocation orthodontique.

Les instruments de parodontologie sont faits d'acier carbone ou chirurgical et parfois les inserts sont faits de carbures de tungstène fondus à la base en acier. De nombreux instruments endodontiques manuels et mécanisés (limes, broches, râcleurs) sont en acier inoxydable austénitique; de récents instruments endodontiques sont en alliages NiTi.

Les alliages non précieux voient leur application en implantologie avec les implants sous-périostés en $\mathrm{CoCr}$ de grade chirurgical et autres alliages à base cobalt. Une nouvelle classe d'acier austénitique a été Iso-standardizée ISO 5832-9. L'acier inoxydable, se corrodant in vivo, est implanté temporairement: plaques d'ostéosynthèse et vis. Les alliages de titane sont principalement utilisés en implantologie.

Des instruments chirurgicaux, instruments de dentisterie ainsi qu'instruments rotatifs (contre-angle, turbine...), sont faits d'aciers inoxydables ou d'alliages $\mathrm{NiCr}$.

Le choix de l'alliage non précieux pour les prothèses diffère dans le monde; tandis que quelques pays privilégient des alliages $\mathrm{Ni}-\mathrm{Cr}$ avec ou sans béryllium, les plus utilisés en Europe et au Japon sont actuellement les alliages $\mathrm{Co}-\mathrm{Cr}$ et les alliages contenant plus de $1 \%$ de nickel ne sont plus utilisés en général. Des inquiétudes sur la biocompatibilité des alliages $\mathrm{NiCr}$ et $\mathrm{NiCrBe}$ ont même mené à leur interdiction dans certains pays européens. En Suède, titane et alliages Co-Cr sont les plus populaires substituts de l'or [15]. Depuis les années 1980, les alliages NiTi prédominent en orthodontie. Les inquiétudes sur l'allergénicité du nickel ont conduit récemment à l'introduction de nouveaux aciers inoxydables (fils pour arcs ou brackets), sans ou avec un très faible contenu en nickel, qui présentent une meilleure résistance à la corrosion, une plus forte dureté et logiquement un faible potentiel allergénique $[1,2]$.

25 éléments environ peuvent entrer dans la composition des alliages dentaires non précieux. Le composant majoritaire ( $\mathrm{Ni}, \mathrm{Cr}$, Co et $\mathrm{Fe}$ ) appelé base (ou solvant) permet de classer les éléments. Les métaux minoritaires (éléments alliés ou soluté), sont ajoutés en faibles proportions afin d'améliorer leurs propriétés. Les compositions pondérales sont variables selon les fabricants. La composition chimique des alliages dentaires coulés non précieux commercialisés répond à des normes internationales.

La composition chimique des alliages dentaires coulés non précieux commercialisés répond à aux normes internationales :

I NF EN ISO 1562 (avril 1995 alliages dits «alliages conventionnels »)

I NF EN ISO 8891 et NF EN ISO 6871-1 (alliages dentaires non précieux à couler à base de cobalt ; avril 1997)

I NF EN ISO 6871-2 (alliages dentaires non précieux à couler à base de nickel, avril 1997)

I NF EN ISO 16744 (matériaux métalliques non précieux pour restaurations dentaires fixées)

I NF EN ISO 9693 (décembre 2000 ; métaux et alliages dentaires pour prothèses céramométalliques),

\ISO TC 106/SC2 N (matériaux métalliques pour restaurations et dispositifs dentaires fixes et amovibles) $\checkmark$ NF EN ISO 22674, mars 2007 : matériaux métalliques pour restaurations fixes et amovibles et appareillages).

NF EN ISO 5832-1 à 12 (matériaux d'implants chirurgicaux (alliages base cobalt et aciers inoxydables).

Selon les normes ISO 6871 part. 1 et 2 tous les éléments de concentration supérieure à $0,1 \%$ doivent être précisés par le fabricant. Ces alliages ne peuvent contenir plus de $0,02 \%$ de béryllium.

\begin{tabular}{|c|c|c|} 
& $\begin{array}{c}\text { Alliages } \\
\text { base } \\
\text { COBALT }\end{array}$ & $\begin{array}{c}\text { Alliages } \\
\text { base } \\
\text { NICKEL }\end{array}$ \\
\hline Norme & ISO 6871-1 & ISO 6871-2 \\
\hline Molybdène & $\geq 4 \%$ & $\geq 4 \%$ \\
\hline Chrome & $\geq 25 \%$ & $\geq 20 \%$ \\
\hline $\begin{array}{c}\text { Cobalt + Chrome } \\
+ \text { Nickel }\end{array}$ & $\geq 85 \%$ & $\geq 85 \%$ \\
\hline
\end{tabular}

I Les alliages base nickel (Ni-Cr, Ni-Cr-Fe, $\mathrm{Ni}-\mathrm{Cr}-\mathrm{Mo}$, Ni-Cr-Co-Mo) sont essentiellement composés de nickel : 60-80\% en poids (voire $15-54 \%$ pour certains arcs orthodontiques) et de chrome : $10-26 \%$. Ils sont souvent riches en fer (10-12\%) ou en molybdène (0-10\%), avec des éléments mineurs $(0,1$ à $14 \%)$. Leurs difficultés de finition et de polissage ont restreint leur emploi. Certains laboratoires choisiraient des alliages de palladium pour sauver les coûts lors de la confection de couronnes ou bridges.

I Les alliages CoCrMo, CoCrW contiennent 50-70\% de cobalt et 20-30\% de chrome. Les autres alliages base cobalt contiennent moins de cobalt et de chrome mais plus de nickel ou de tungstène. L'alliage Elgiloy ${ }^{\circledR}$ (bagues, fils, arcs) est un alliage 
CoCr $(40 \% \mathrm{Co}, 20 \% \mathrm{Cr}, 15 \% \mathrm{Ni}, 7 \% \mathrm{Mo},<2 \%$ $\mathrm{Mn},<0,1 \% \mathrm{C}, 0,1 \% \mathrm{Be}, \mathrm{Fe}$ balance). Devant les difficultés de mise en œuvre du titane et ses alliages, les fabricants recherchent de nouveaux alliages base cobalt plus résistants à l'abrasion : essentiellement alliages Co-Cr où nickel, fer, béryllium, gallium et cuivre sont éliminés. Quelques nouveaux alliages CoCr, références pour la prothèse amovible coulée (Remanium $^{\circledast}$, Cerabalt $^{\oplus}$, Wironit ${ }^{\circledast}$, Wironium ${ }^{\circledR}$ ) ont globalement amélioré leurs propriétés physiques, mécaniques et biologiques

I Les aciers inoxydables médicaux (AISI 316L, AISI 303, ASTM F-55 et F-138) contiennent 17-20\% Cr, $13-15 \% \mathrm{Ni}, 2-3 \% \mathrm{Mo}$, Fe (balance) et parfois des impuretés : barium, strontium, cobalt, plomb. De nouveaux aciers inoxydables austénitiques contiennent moins de nickel (Tab. I) ; les fils « Truchrome » et fils australiens contiennent respectivement 8 et $8,9 \%$ de nickel.

I Titane et alliages de titane 4 grades de titane commercialement pur (Ti-cp) se distinguent en fonction du pourcentage de différents éléments (oxygène, fer, carbone, azote, hydrogène). D'autres éléments (aluminium, vanadium, palladium, nickel ou cuivre) peuvent être ajoutés en quantité plus importante, réalisant un alliage de titane. En implantologie, seul le TA6V ( $\mathrm{Ti}-6 \% \mathrm{Al}-4 \% \mathrm{~V})$ est employé. Les alliages à mémoire de forme « $\mathrm{Ni}-\mathrm{Ti}$ » ou « Nitinol » sont employés principalement en orthodontie (arcs, fils) mais aussi endodontie. Les limites de leurs compositions selon la norme ASTM sont : nickel 54,5-57 \%, C, Co, Cu, Cr, H, Fe, Nb, O $\leq 0,25 \%$ et balance : titane. L'alliage TMA (Titane Molybdène Alloy) des fils d'orthodontie, contient 77,8\% de titane, $11,3 \%$ de molybdène, $6,6 \%$ de zirconium et $4,3 \%$ d'étain.

\section{MÉCANISMES DE L'ALLERGIE}

L'allergie peut être définie comme une manifestation anormale du conflit antigène-anticorps après une première phase de sensibilisation. Les interactions biologiques d'alliages dentaires coulés avec le tissu oral ne sont pas totalement comprises (spécialement les différences individuelles) [3]. La réponse biologique d'un alliage dépend de la nature, quantité et forme de l'élément libéré (particules ou ions complexés ou libres hydratés) et de la durée d'exposition des cellules et tissus. Des ions inactifs plus longtemps dans un état ionique ont plus de chance de se combiner avec des biomolécules et révéler une toxicité ou allergie que des ions actifs qui se combinent immédiatement pour former oxydes, hydroxydes ou sels inorganiques. Des réactions d'hypersensibilité de type I (ou immédiate; tel l'urticaire ou l'angio-oedème) (Fig. 1) peuvent être initiées par des sels de chrome et de nickel mais pas directement à partir de biomatériaux implantés [4]. Chez un individu sensibilisé, les manifestations cliniques d'une hypersensibilité immédiate apparaissent 6 à 12 heures après le contact avec l'allergène et atteignent un maximum à 24,48 voire 72 heures. Nickel, chrome ou cobalt deviendraient allergéniques quand ils sont combinés avec des protéines in vivo (albumine...) par des mécanismes médiés par IgE métal spécifique. Mais d'autres métaux (aluminium, zinc, vanadium) induiraient des asthmes occasionnels par des mécanismes non médiés par IgE [16]. La majorité des réactions allergiques aux alliages dentaires (notamment nickel [5]) sont des réactions d'hypersensibilité de type IV (ou retardée) [3, 1]. Elles se manifestent par une allergie de contact [6] avec réaction locale [17] et peuvent prendre des mois à des années pour se développer [4].

L'allergie n'est pas dose-dépendante [7]; chez un individu sensibilisé les réponses allergiques peuvent être initiées par de petites quantités d'allergènes [1]. Le risque d'hypersensibilité augmente avec la fréquence d'exposition au métal. Des ions métalliques (sels) doivent être relargués à partir d'un alliage pour causer une allergie car celui-ci ne peut être allergène par lui-même. Ils agissent plutôt comme haptènes, se complexant à des protéines, acides nucléiques ou carbohydrates, possédant alors un fort potentiel allergénique [7]. La porte d'entrée de l'allergène peut être cutanée (périorale), muqueuse, digestive (absorption

\begin{tabular}{|l|c|c|c|c|c|c|c|c|c|} 
& Fe & Ni & Cr & Mn & P & Si & S & C & Mo \\
\hline AISI 304L & Bal & 7,37 & 19,50 & 1,32 & 0,04 & 0,48 & 0,01 & 0,03 & 0,31 \\
\hline SUS 316L & Bal & $10,00-14,00$ & $16,00-18,00$ & $<2,00$ & $<0,04$ & $<1,00$ & $<0,03$ & $<0,08$ & $2,00-3,00$ \\
\hline SUS 303 & Bal & $8,00-10,00$ & $17,00-19,00$ & Max 2,00 & & Max 1,00 & & & 0,60 \\
\hline SUS 302 & Bal & $8,00-10,00$ & $17,00-19,00$ & & Max 0,05 & Max 1,00 & Max 0,03 & Max 0,15 & \\
\hline AISI 4340 & Bal & 1,46 & 0,80 & 0,69 & 0,01 & 0,34 & 0,01 & 0,42 & 0,22 \\
\hline
\end{tabular}

$\triangle$ Tableau I : Composition d'aciers inoxydables commercialisés (Ni-Cr-Fe ; \% poids). 


\begin{tabular}{|l|c|c|c|c|c|c|c|c|c|c|} 
& Co & Cr & Mo & Si & Mn & C & Fe & Ce & W & Autres \\
\hline Wirobond LFC $^{\circ}$ & 33,0 & 30,0 & 5,0 & 1,0 & 1,5 & 0,3 & 29,0 & & & N 0,2 \\
\hline Neobond II $^{\circ}$ & 52,2 & 27,8 & & 0,5 & & 0,4 & 0,5 & & & Ga 2,5 \\
& & & & & & & \\
\hline Remanium R2000 $^{\circ}$ & 61,0 & 25,0 & 7,0 & 1,5 & $<1,0$ & $<1,0$ & & $<1,0$ & 5,0 & \\
\hline Wirobond C+ $^{\circ}$ & 61,5 & 26,0 & 6,0 & 1,0 & & & 0,5 & & 5,0 & \\
\hline Vitallium $^{\circ}$ & $60,6-62,5$ & $30,8-31,5$ & $5,1-6,0$ & $0,3->1,0$ & $0,5->1,0$ & $0,4->1,0$ & 0,7 & & & \\
\hline Remanium GM800 $^{\circ}$ & 63,5 & 30,0 & 5,0 & 1,0 & 0,2 & 0,3 & & & & \\
\hline Wironit & 64,0 & $28,5-28,6$ & 5,0 & $<0,35-1$ & $<0,35-1$ & $<0,35-0,5$ & & & & \\
\hline
\end{tabular}

$\triangle$ Tableau II : composition de quelques alliages dentaires CoCr commercialisé pour la technique céramo-métallique en prothèse conjointe et pour châssis de prothèses amovibles (\% poids).

de particules) ou respiratoire (inhalation de poussières, vapeurs). La réponse allergique peut apparaitre localement (réaction cutanée par simple contact : dermatite ou stomatite de contact et eczéma) ou à distance du tissu en contact avec l'allergène [17] après un trajet indirect (salive, intestin, sang et peau/muqueuse); les manifestations à distance, respiratoires ou cutanées peuvant être concomittantes. Les cas d'allergie attribuées aux alliages dentaires non précieux sont rares [3]. Peu de réactions allergiques intraorales sont décrites même si les individus sont sensibles [1] et malgré l'utilisation intraorale de composants contenant un allergène connu. Les manifestations orales d'allergies aux métaux sont de diagnostic difficile [5] et plus rares que les réactions cutanées [1], notamment du fait d'une moindre susceptibilité à former des antigènes ou allergènes après couplage avec un ion métallique. Ceci peut s'expliquer aussi par le fait qu'en général, les muqueuses orales sont plus résistantes $[18,22]$ et moins réactives [1] que la peau à une irritation primaire et ne sont pas aussi facilement sensibilisées [18, 22], peut être à cause des effets irrigants et tampons de la salive (qui lave des sensibiliseurs de la muqueuse) et à des contenus plus faibles de protéines porteuses des allergènes [8]. Les cellules de Langerhans sont moins nombreuses que dans la peau. Des concentrations plus fortes d'allergènes seraient peut être nécessaires en bouche pour montrer des manifestations allergiques [5]; il faut notamment 5 à 12 fois plus de nickel en bouche qu'en contact avec la peau. De plus les produits relargués des matériaux dentaires sont dissouts dans la salive, relargués dans une forme inactive ou avalés avant de réagir ou être absorbés [1]. Une vascularisation de la muqueuse orale abondante permet aussi une absorption rapide et un nettoyage des allergènes [18]. Les alliages dentaires sont aussi plus résistants à la dégradation que la plupart des autres alliages employés quotidiennement.

\section{MANIFESTATIONS ORALES ET EXTRAORALES}

Les dermites de contact sont les exemples les plus classiques de manifestations cliniques des réactions allergiques aux alliages non précieux [18]. Les signes cliniques sont indépendants de la durée d'exposition aux métaux [5]. Des réactions apparaissent à des sites extraoraux dans des aires de contact avec des bijoux en acier inoxydable ou plaqués chrome avec eczéma et prurit sous forme d'un erythème local parfois papulaire ou vésiculopapulaire dans la phase chronique [17].

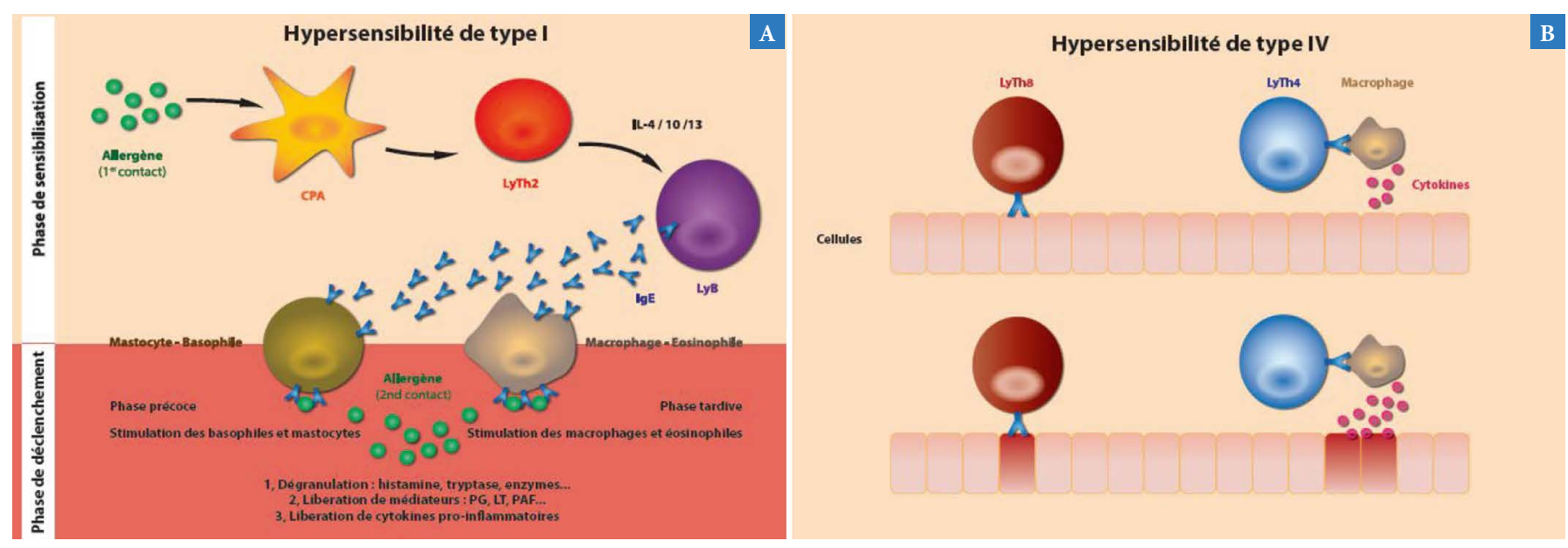

$\triangle$ Figure 1 : a) mécanismes de l'hypersensibilité de type I ; b) mécanismes de l'hypersensibilité de type IV. 
Les manifestations orales d'allergie aux alliages dentaires sont rares et de diagnostic difficile [5] car peu spécifiques. Il est difficile voire impossible de différencier une lésion buccale provoquée par une allergie de celle provoquée par une intolérance, la corrosion ou une mycose par exemple. L'effet le plus commun des réactions allergiques orales aux alliages dentaires est l'allergie de contact [6] : stomatite allergique avec réactions érythémateuses, œdémateuses, vésiculaires, érosives, bulleuses ou ulcérées. Citons aussi les lésions blanches limitées à la surface de contact, les réactions lichénoïdes [3] de la muqueuse orale et de la gencive [7], les kératoses [3], des gingivites persistantes, une glossite, une chéilite, un rash cutané, un oedème, des douleurs, une nécrose locale des tissus mous ou osseux conduisant à la perte d'implants [4] ou une hypertrophie et hypersensibilité des papilles linguales. Les signes subjectifs (douleurs, prurit, sensation de brûlures, de picotements, dysesthésies ou paresthésies de la muqueuse buccale, labiale ou linguale en contact avec le métal...) sont souvent plus importants que les signes objectifs.

Le risque allergique immédiat avec les alliages dentaires à base de nickel serait celui de dermatites de contact. Les châssis de prothèses amovibles même avec un contenu faible en nickel ( $1,5 \%$ en poids) peuvent en être à l'origine [8]; leur corrosion semblerait aussi mener à l'inflammation des tissus mous (stomatite) et à une dermatite de contact. Des miniplaques d'ostéosynthèses en acier inoxydable font état d'hypersensibilité retardée au nickel et des implants et couronnes en alliages riches en nickel ont fait état de pertes significatives d'os alvéolaire [8]. Les réactions allergiques au nickel et à ses dérivés apparaissent en bouche en contact et à proximité de structures métalliques à base de nickel. Leurs signes et symptômes incluent : gingivite, hyperplasie gingivale, œdème, desquamation labiale, lèvres fissurées, chéilite angulaire, stomatite, sensation de brûlure de la muqueuse orale mais aussi

manifestations extra-orales (rashs eczémateux) [5,1]. Les manifestations de l'allergie aux alliages dentaires peuvent apparaître à distance des métaux intra-oraux : elles sont plus fréquentes et parfois concomittantes. Les principales sont des réactions cutanées : dermite, dermatite, urticaire, eczéma (ulcération possible), sensibilités, œdème des paupières, lèvres fissurées, eczéma chronique des joues et paumes des mains, urticaires de contact et plaques érythémateuses voire oedémateuses. Les dermatites se situent généralement à distance sur les mains et les avant-bras ou peuvent être généralisées avec une prédilection de localisation et un eczéma atopique au niveau du scalp, des paupières, des oreilles et des lèvres [9]. Des réactions respiratoires (rhinites, laryngite, bronchites, asthme), gastroentérologiques (oesophagites, gastro-entérites) ou ophtalmologiques (conjonctivites) peuvent apparaître.

La dissémination systémique des débris métalliques dentaires (directement ou indirectement par les macrophages) pourraient causer des dommages à distance. L'ingestion de quantités appréciables de métaux à partir de l'alimentation et des alliages dentaires chez des personnes sensibilisées, peut induire des dermatoses ou exacerber certaines dermatites (eczéma discoïde...): dermatite des mains, lichen plan, pustulose palmaire et plantaire. La corrosion chimique ou électrochimique est responsable de réactions buccales parfois difficile à différencier d'une réaction allergique. Les alliages et ions métalliques peuvent avoir un potentiel local toxique et induire une inflammation [7] et une irritation des tissus cutanées ou oraux adjacents [2] avec des lésions des muqueuses, hypersensibilité, inflammation et nécrose tissulaire. Ils stimuleraient l'ostéolyse périprothétique et génèrent souvent une réponse inflammatoire et immunologique des tissus péri-implantaires suggérant qu'ils pourraient contribuer à des échecs implantaires [10]. Des inflammations sous les prothèses amovibles à châssis métallique sont inquiétantes mais en rien le constat d'une réaction allergique, elles sont souvent le témoignage d'une stomatite réactionnelle à une contamination bactérienne ou fongique des prothèses, à une perte d'adaptation physique et mécanique des prothèses (Fig. 2) ou à une réaction à la corrosion des composants métalliques. Modifications et réparations (soudures, brazures) des prothèses et appareils orthodontiques peuvent favoriser la corrosion des structures métalliques et seraient à l'origine de réactions inflammatoires et irritation des tissus mous mettant en évidence la nocivité des produits de corrosion.

Avec une intolérance à l'alliage, les manifestations peuvent apparaître le jour même, la semaine ou le mois qui suit l'installation du polymétallisme. Déposer le ou les éléments anodiques, cause d'électrogalvanisme, permet la guérison [3].

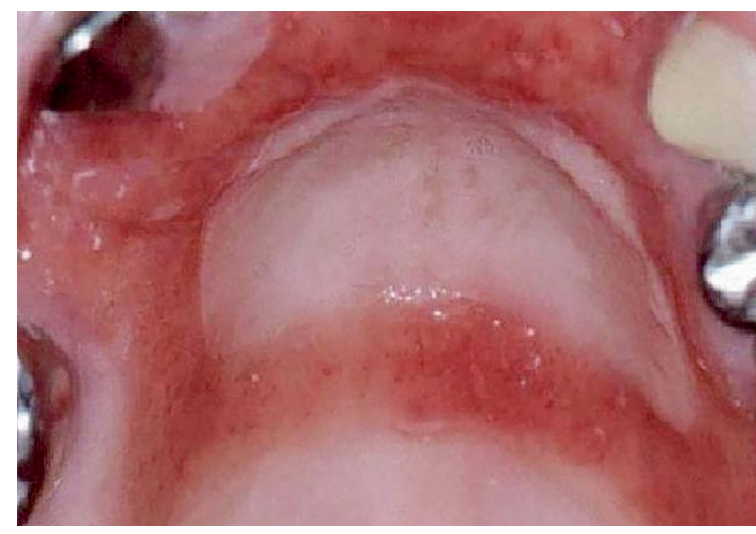

$\triangle$ Figure 2. 


\section{INFLUENCE DE LA NATURE DU METAL}

La fréquence d'hypersensibilité aux ions métalliques diffère considérablement parmi les métaux, ceci probablement dû à la fréquence d'exposition de la population à ces métaux [7], mais une composante génétique interviendrait aussi. La forme chimique du métal influence aussi le risque de sensibilisation.

Des réactions allergiques modérées ou sévères apparaissent avec des alliages dentaires non précieux principalement alliages $\mathrm{NiCr}$ et $\mathrm{CoCr}$ [3]. Les alliages base nickel et leurs produits de corrosion présentent une toxicité et un potentiel de sensibilisation et d'allergie [1,5] plus grands que les alliages CoCr dont les allergies sont rares [15]. Nickel, chrome et à moindre degré cobalt, or et palladium sont reconnus comme les métaux les plus sensibilisants; béryllium, cuivre, molybdène, titane, mercure, étain, zinc et platine [1,7], argent, indium, irridium, fer, manganèse sont aussi à l'origine de réactions allergiques. Selon les études, entre 15 et $18 \%$ de la population est reconnue sensible au nickel, entre 8 et $15 \%$ au cobalt et entre 3,5 et $8 \%$ au chrome [4,7]. Les hommes sont moins sensibles et réagissent moins souvent au nickel que les femmes mais beaucoup plus au chrome [9]. Une allergie croisée aux métaux est possible [7]. La cosensibilisation au nickel, cobalt et chrome apparaît fréquente. Il a été suggéré que la sensibilisation au nickel et une dermatite préexistante sont souvent des prérequis pour une sensibilisation au cobalt [9]. Une réaction allergique croisée a été mise en évidence pour le palladium et le nickel [7,3]. Les patients sensibles au palladium se montrent presque toujours sensibles au nickel [7]. Cette réaction n'intervient que dans le cas où la première sensibilisation a été faite avec du palladium sous forme de chlorures. 34 à $64,5 \%$ des patients allergiques au nickel le seraient aussi aux ions palladium et seule une faible proportion de ceux-ci réagit à la forme métallique du palladium.

Le nickel est le composant métallique principal de nombreux alliages dentaires. Le potentiel allergénique du nickel, soluble dans le serum et les liquides biologiques, apparaît douteux pour Arikan A ; Fisher a même suggéré que le nickel est tellement lié dans l'alliage que les liquides biologiques ou la perspiration ne peuvent le libérer et lui permettre de produire des réactions allergiques [8]. Pour d'autres, c'est un allergène modéré et les patients ne semblent pas avoir de risque significatif de développer une sensibilité au nickel seulement attribuable au contact d'un matériau dentaire contenant du nickel [1]. Il est dit que la présence chez des patients allergiques au nickel d'une forte concentration de nickel dans les alliages dentaires n'est pas nécessairement associée à une stomatite de contact allergique [8]. Tous les patients sensibilisés au nickel ou à ses sels ne montreront pas de réaction allergique [5] mais la probabilité est supérieure [3] même si elle reste assez rare [5]. Les risques de réactions locales d'hypersensibilité sont réels par contre les signes cliniques seraient indépendants de la durée d'exposition [5]. Les absorptions cellulaires de nickel pourraient aussi être indépendants des concentrations sériques [8].

Par contre d'après Mockers $\mathrm{O}$ et al, les alliages à base de nickel font partie des matériaux orthodontiques les plus allergènes [19]. Le nickel y est d'après Sakai Y et al le plus fréquemment en cause du fait de sa facile ionisation [17]. Le nickel par contre est faiblement sensibilisant sous forme de chlorures. Il est dit fortement allergène $[8,20]$ par lui-même ainsi que ses sels ; il serait même le plus allergisant de tous les éléments métalliques selon d'autres auteurs et responsable de plus de réactions allergiques, dermatites de contact et eczéma des mains $[6,11,12]$. Des concentrations tissulaires élevées d'ions nickel causeraient les réactions allergiques de contact les plus étendues [12].

Le chrome n'est pas sensibilisant par lui-même mais au contact de la sueur, de la peau, il se forme un sel de chrome ; les sels et les dérivés ((bi)chromates) ont un potentiel allergique et toxique [21]. Le chrome hexavalent $\left(\mathrm{Cr}^{6+}\right)$ est un allergène fréquent [12]. Les dérivés trivalents du chrome $\left(\mathrm{Cr}^{3+}\right)$ sont des haptènes et peuvent se complexer à une protéine initiatrice de sensibilisation mais cette sensibilité, très rare, nécessite des concentrations élevées. Les intoxications chroniques sont liées soit au contact direct du métal ou ses dérivés avec la peau ou les muqueuses soit à l'inhalation des dérivés.

Le cobalt est un fréquent allergène [12], avec selon les études, une sensibilité identique pour les deux sexes ou majoritaire pour les femmes [9].

\section{ALLERGIE AU NICKEL : DONNÉES DE LA LITTÉRATURE}

À l'inverse de Newman, d'après Setcos JC et al, il n'est pas évident d'affirmer que les patients ont un risque significatif de développer une sensibilisation ou réaction allergique par le seul contact permanent de la surface d'une couronne à base de nickel avec les liquides oraux [1]. D'après la littérature, les réactions d'hypersensibilité au nickel apparaissent vraisemblablement seulement après une sensibilisation primaire par un contact non dentaire [1]: contact de la peau direct et prolongé avec des objets qui relarguent des ions nickel [6]. Généralement, il s'agit d'une exposition domestique quotidienne ou fréquente aux robinetteries, ustensiles ménagers, lessives, boutons de jean, 
fermetures éclair et attaches de vêtements et sous-vêtements mais aussi par l'atmosphère, les aliments et l'eau de boisson [8]. Le port de boucles d'oreilles, bijoux, bracelets de montres ou l'effraction du revêtement cutané sont également des facteurs de risque de sensibilisation au nickel [7], reconnus comme les causes majeures de sensibilisation chez des patients non allergiques et de dermatites de contact allergiques chez des individus déjà sensibilisés [6].

L'incidence de réactions adverses face aux alliages dentaires contenant du nickel est faible [11], estimée de 1 pour 100 patients traités par dispositifs orthodontiques dont $85 \%$ attribués à des dermatites de contact $[3,5]$. Cette incidence passe à 1 pour 330 patients traités par prothèses [3]. La prévalence et l'incidence d'allergies et d'eczémas des mains induits par le nickel chez les femmes et les hommes adultes serait en Europe de $10-15 \%$ et 1-3\% respectivement selon les études $[6,11,12]$ et environ 30-40\% des personnes sensibles rapportent de l'eczéma des mains [6]. Ceci est probablement le résultat d'une plus forte et plus fréquente exposition au nickel des femmes.

Adolescents et jeunes adultes tendent à avoir une prévalence plus forte [12]; chez les 14-18 ans : $30 \%$ des filles et $3 \%$ des garçons. Cette sensibilisation au nickel plus forte est expliquée par le perçage des oreilles et le port de boucles d'oreilles vu que la prévalence de sensibilité au nickel chez les filles avec les oreilles percées est de $31 \%$ contre $2 \%$ sans. Dans plusieurs études cliniques, aucune des filles traitées avec un appareil orthodontique fixe contenant du nickel avant perçage des oreilles n'a montré une hypersensibilité au nickel ; mais $35 \%$ des filles avec les oreilles percées avant le traitement orthodontique étaient sensibles au nickel $[1,3,11]$. Ce sont donc généralement les patients avec une présensibilisation au nickel par les boucles d'oreille, portées à un jeune âge, qui après insertion d'un appareil orthodontique à base de nickel développent une dermatite de contact allergique [5,1]. Par contre, ce traitement orthodontique à base de nickel avant sensibilisation au nickel par le perçage des oreilles et port de boucles d'oreilles apparaît réduire la fréquence d'hypersensibilité au nickel $[3,11,1]$. Il est affirmé aussi que l'utilisation intraorale de composants contenant un allergène connu, comme le nickel, pourrait améliorer ou induire une tolérance immunologique à cet allergène chez des patients sensibles ou non au nickel [1,5], pourrait réduire le degré de sensibilisation de patients reconnus allergiques et s'avérer favorable à une désensibilisation. Des questionnaires ont conclu que les personnes sensibles au nickel n'ont pas un risque élevé de développer des réactions systémiques et orales, ou exacerbation des lésions de la peau après une exposition orale au nickel [1]. D'autres auteurs suggèrent au contraire que les traitements orthodontiques sensibilisent les patients [11].

\section{DÉPISTAGE ET PRISE EN CHARGE DE L'ALLERGIE}

La prise en charge d'un patient (potentiellement) allergique passe d'abord par le dépistage de l'éventuelle allergie. Avant de poser l'indication d'un alliage, un interrogatoire préalable du patient sur ses antécédents personnels et familiaux (terrain allergique avec dermatites de contact...), une anamnèse et un examen clinique rigoureux doivent être entrepris [17]. L'allergie aux métaux dentaires, certainement surestimée, doit être recherchée devant des manifestations endobuccales cliniques évocatrices, après avoir éliminé les autres causes [3] mais cliniquement, en intra-oral rien ne permet de distinguer une simple irritation d'une allergie et la disparition des signes cliniques et subjectifs (douleurs...) à l'ablation du matériel incriminé n'est pas une preuve suffisante. Des tests dermatologiques, si nécessaires, devront faire le diagnostic différentiel entre allergie et intolérance et déterminer l'allergène responsable. Quand l'allergie sera avérée, l'éviction (si possible) des métaux incriminés devra être entreprise.

L'allergie ou hypersensibilité à un métal est validée avec des tests épicutanés («patch-tests»), pas toujours fiables, ou en injectant ce métal sous la peau [7]. Seuls des tests muqueux permettraient de résoudre les cas douteux. Des « prick tests » dépistent les allergies de type I. Chez des patients présentant des réactions d'hypersensibilité violentes, seront privilégiés des tests in vitro du type TTL (test de transformation lymphoblastique) pour compléter le patch test [8]. Des publications ont suggéré qu'une exposition à long terme à des alliages dentaires à base de nickel peut affecter les monocytes et les cellules des muqueuses orales [1] avec une augmentation significative de la transformation des lymphocytes. Toutefois un test dermatologique de dépistage des allergies n'est pas recommandé par tous à cause d'une possible sensibilisation [3].

Il est recommandé d'éviter des alliages dentaires à base des éléments dont l'hôte est reconnu sensible $[4,7,12]$ et préférer des matériaux résistants à la corrosion. S’il s'agit du nickel, chrome ou cobalt, il est recommandé de privilégier des alliages précieux, titane, alliages de titane, céramiques et résines. Le titane sera préféré pour la prothèse fixe et les châssis de prothèse adjointe. Si une allergie au nickel est reconnue, il faut employer des alliages sans nickel ou palladium mais le choix est restreint [17]. 
La suppression des restaurations contenant les métaux incriminés amène généralement la disparition rapide et complète des signes pathologiques associés (cliniques et douloureux). La dermatite associée se résoud généralement entre 2 jours et plusieurs mois après retrait [1]. On peut constater en cas d'allergies au cobalt ou chrome, quelques jours à quelques semaines après début de port d'une prothèse adjointe partielle en Co-Cr des symptômes et le port continu peut devenir intolérable. Les symptômes douloureux et signes muqueux, signes d'hypersensibilité cessent progressivement après retrait de la prothèse.

\section{MESURES PRÉVENTIVES POUR LES PATIENTS}

Pour ne pas créer de sensibilisations ultérieures, il faut limiter la libération d'ions métalliques (générée par une dégradation mécanique, chimique ou électrochimique). Plusieurs recommandations pratiques et précautions à prendre peuvent être retenues au niveau du fabricant, du prothésiste et du praticien dans la sélection de l'alliage.

Il est recommandé un traitement irréprochable des alliages au laboratoire : éviter les défauts de fonderie, choix des creusets, maîtriser les procédures de refroidissement, polir parfaitement les surfaces et prévoir un traitement thermique final de réhomogénéisation. La recoulée des alliages $\mathrm{NiCr}$ et $\mathrm{CoCr}$ ou l'emploi d'alliages $\mathrm{NiCr}$ à base de cuivre (Thermobond ${ }^{\circledR}$ ) fragiliserait leur résistance à la corrosion.

Il est important de réduire les phénomènes d'électrogalvanisme en bouche pour prévenir les risques de corrosion ; cela passe par le choix de matériaux résistants à la corrosion et d'alliages à microstructure monophasée. Les restaurations doivent être les plus homogènes possibles; il faut éviter le polymétallisme, privilégier les prothèses composites homométalliques. Il faut connaître les compositions exactes et les traitements mécaniques et thermiques des éléments, relever la présence d'un piercing, éliminer les éléments responsables de signes cliniques. Le praticien doit choisir le même alliage, la même famille ou au moins une famille d'alliages compatibles avec l'alliage présent, proches dans la série galvanique, inertes [22], de préférence avec le plus bas potentiel de corrosion. Les alliages CoCr et à base titane ont un comportement "noble " sur le plan électrochimique. De même, si la composition chimique du châssis d'une prothèse amovible est inconnue, il est recommandé d'éviter de la réparer et de procéder à son remplacement pour éviter tout couple galvanique à l'origine de corrosion.
Les alliages de brazage notamment relarguent des éléments cytotoxiques : cadmium, cuivre et zinc [2].

\section{MOYENS DE PROTECTION DES PROFESSIONNELS}

Les alliages dentaires non précieux (notamment alliages $\mathrm{NiCr}$ ) peuvent être à l'origine d'affections professionnelles. Les dermites de contact induites par leurs sels sont inscrites au tableau des maladies professionnelles.

Les techniciens des laboratoires de prothèses sont exposés au risque d'intoxication ou sensiblisation/ allergie par inhalation de poussières, vapeurs ou gaz générés par les travaux de coulée, dégrossissage, usinage et finition des pièces prothétiques. Le chirurgien dentiste y est aussi confronté lors du fraisage des pièces métalliques. Les particules d'alliages libérées [16] peuvent induire des pathologies cutanées (irritations et allergies de contact : eczéma de contact) et pulmonaires (pneumoconioses, pneumopathie d'hypersensibilité, syndromes d'irritations bronchiques, asthme, trachéite, rhinite, fibrose, cancer, mésothéliome et collagénose [1]). Le béryllium des alliages $\mathrm{Ni}-\mathrm{Cr}$ et $\mathrm{Co}-\mathrm{Cr}$, toxique, est à l'origine de pathologies sévères (bérylliose et cancer) et manifestations allergiques (dermites, dermatoses, rhinites, conjonctivites et bronchites) [13]. A la suite des études de Moffa, certains alliages contenant du béryllium ont été retirés du marché mais ont fait un retour en force avec le développement des bridges collés, car ce sont les seuls à posséder une réelle aptitude au mordançage. Les moyens de protection mis en place doivent évoluer avec l'accroissement des risques. Ils visent à protéger les poumons et à un dépistage précoce. L'utilisation de microtours actuels plus puissants est responsable d'une poussière plus fine et abondante. Les mesures précisées par la réglementation, portent sur la suppression ou limitation des poussières et vapeurs par l'utilisation de nouveaux produits ou techniques (préférer la fusion par induction haute fréquence), par un système de ventilation locale ou de captage des poussières au plus près des sources d'émissions, ventilation générale, salles spécifiques, isolement des sources de pollution, stockage des produits dangereux, ventilation interne aux locaux, hotte [1] et aspiration standard. Il est recommandé d'effectuer les finitions sur tour fixe horizontal plutôt qu'à la pièce à main, d'employer un air de compensation, rejet extérieur ou recyclage de l'air capté et précautions simples (équipement de protection individuelle: écran, masque, filtres de classe P2, lunettes, aspiration des particules sur place). Le 
ministère chargé du travail a adopté de nombreuses valeurs limites d'exposition pour certains polluants à ne pas dépasser. La prévention des risques professionnels s'appuie sur les principes édités par les articles L.4121-1 à L.4121-5 du code du travail [14].

\section{ACTUALITÉS, LÉGISLATION ET SOLUTIONS}

De nombreux alliages non précieux sont disponibles sur le marché. Les fabricants doivent garantir que les caractéristiques spécifiques des dispositifs médicaux sur mesure sont en conformité avec les exigences de la directive européenne "Dispositifs médicaux » 93/42/CEE du 14 juin 1993, depuis la conception jusqu'au contrôle final, exigences qui fixent les modalités de mise sur le marché [14]. Le distributeur doit se conformer à ces exigences. Choix et évaluation clinique de tout matériau ou dispositif médicaux destinés à l'homme sont réglementés par cette directive ainsi que la directive « dispositifs médicaux implantables actifs » 90/385 CEE. La directive 93/42/ CEE précise que le prothésiste ne doit utiliser que des alliages ou produits conformes aux normes françaises et européennes ou Iso [14]. La transposition en droit français de cette directive (Article 14 notamment) a été réalisée dans le livre II du titre $\mathrm{I}^{\text {er }} \mathrm{du}$ code de santé publique en vigueur depuis le 14 juin 1998 : Article R.5211-65 et R.5211-70. La directive 93/42/ CEE a été révisée en 2007 par la directive 2007/47/CE. Les fabricants doivent vérifier que le produit répond à la définition de dispositif médical (art. L.5211-1 et R.5211-4 du CSP) sur mesure décrite à l'article R.5211-6. La mise sur le marché est soumise aux procédures de marquage $\mathrm{CE}$ au titre de la directive 93/42/CEE, authentification du certificat de marquage transmis par le fabricant. Conformément à la directive 93/42/CEE, le praticien est le prescripteur des alliages. Il est responsable du choix des matériaux, de la sûreté et de l'efficacité des restaurations [14]. La sélection d'un alliage ou d'une famille d'alliages homogène repose sur son indication mais aussi des critères fonctionnels (espace disponible, rigidité...), ses propriétés mécaniques, ses possibilités techniques d'élaboration (coulée, brasage, assemblage, usinage, liaison métal-céramique), propriétés physicochimiques (résistance à la corrosion) et biologiques (biocompatibilité) [22, 23], stabilité, longévité, résistance aux contraintes buccales (forces, pressions, variations thermiques...), critères cosmétiques (incidence de la couche d'oxyde de l'alliage sur la teinte de la céramique) et économiques (coût du matériau et de la confection). Le praticien doit s'informer de la composition complète des alliages utilisés par son laboratoire de prothèse et vérifier la fiche technique de l'alliage choisi (marquage CE) ou la fiche de données de sécurité. L'identification (composition, numéro de lot...), le nom de l'alliage et du fabricant sont conservés dans le dossier du patient. Le praticien doit spécifier l'alliage sur la fiche de liaison avec le prothésiste et employer des alliages biocompatibles provenant de laboratoire qualifié, bien informé sur ses alliages. Un certificat de conformité établi par le praticien est fourni au patient pour chaque élément prothétique réalisé. Les informations (notice, étiquetage et déclaration de conformité) données par le fabricant doivent être délivrées au patient avec chaque dispositif médical sur mesure ; elles découlent du point 13 de l'article ler de l'arrêté du 20 avril 2006 relatives aux exigences essentielles de santé et de sécurité. Le fabricant fournit une déclaration selon laquelle le dispositif médical est conforme aux exigences essentielles mentionnées aux articles R.521121 à R.5211-23 du code de la santé publique [14]. Le marquage $\mathrm{CE}$, obligatoire, est gage de sécurité pour le praticien, le laboratoire et le patient, mais pas une marque de certification «qualité » contrairement à la marque NF (certification de l'Agence française de normalisation AFNOR).

Les alliages métalliques dentaires évoluent constamment pour satisfaire aux exigences plus grandes qui leur sont demandées, notamment vers la mise au point d'alliages de meilleure résistance à la corrosion, moindre toxicité avec de nouvelles compositions. Récemment des améliorations de composition des alliages et le développement de nouvelles techniques de fabrication ont optimisé l'utilisation des alliages non précieux. De nouveaux alliages coulés ont été développés en vue d'égaler voire dépasser les propriétés physiques d'alliages précieux à fort contenu en or. En Europe, une législation limite l'utilisation du nickel dans les éléments dentaires [11]. Des recherches ont été menées pour étudier des alternatives valables aux alliages à base de nickel en accord avec les directives EU sur l'utilisation de matériaux à base de nickel pour des «éléments voués à un contact permanent avec le corps humain "; la directive européenne EU Dir. 94/27/CEE, para2) [10] impose un bannissement des éléments manufacturés à base de nickel qui excèdent un taux de relargage de nickel de $0,5 \mu \mathrm{g} / \mathrm{cm}^{2}$ par semaine. Récemment dans une seconde directive 2004/96/EC portant sur le "percing dans le corps humain, la limite du taux de relargage du nickel est de $0,2 \mu \mathrm{g} / \mathrm{cm}^{2}$ semaine [12].

$\mathrm{Du}$ fait de l'allergie au nickel et même si elle est controversée, la tendance est de choisir des alliages sans nickel [24]. Les alliages $\mathrm{Ni}-\mathrm{Cr}$ reconnus à l'origine 
de maladies professionnelles des prothésistes, tendent à être remplacés par des alliages Co-Cr censés être moins allergisants et plus résistants. Aujourd'hui les alliages base cobalt (Co-Cr) seraient les plus employés par les laboratoires en prothèses fixes métalliques ou céramo-métalliques [24].

Pendant plus de cinq décades, les brackets en acier inoxydable avec 8-25\% de nickel ont été les plus utilisés. Les alliages de brazage des brackets sont aussi riches en nickel avec $84 \%$ de nickel [11]. De nouveaux aciers inoxydables austénitiques sans ou avec une quantité réduite de nickel (9-12\% voire moins) $[10,12,21,26]$ et avec un fort contenu en azote, ont été introduits notamment en orthodontie (par exemple pour les brackets), pour tendre à remplacer les aciers inoxydables conventionnels, type 316 et 318 par exemple [2]. Ils ont été récemment développés [10] afin d'améliorer la biocompatibilité des alliages non précieux; ils paraissent avoir des propriétés mécaniques (dureté, résistance) et résistances à la corrosion comparables voire supérieures aux aciers inoxydables conventionnels $[2,10,12]$. Le standard ASTM F222902 désignant un acier inoxydable pauvre en nickel 23 Mn-12-Cr-1Mo renforcé en azote sous forme de barres et fils pour implants chirurgicaux et une nouvelle génération d'aciers austénitiques nommés « sans nickel » stabilisé avec l'azote et le manganèse, sont apparus sur le marché [12]. Un nouvel acier inoxydable austénitique P558 récemment breveté (ASTM E 112 :4-5, contenu élevé en manganèse et azote, négligeable en nickel ( $<0,2 \%$ en poids)) peut fournir une alternative intéressante aux aciers conventionnels, alliages à base cobalt, titane et alliages de titane. Il se montre biocompatible et les processus d'ostéointégration apparaissent significativement améliorés comparés à des aciers inoxydables conventionnels et alliages TA6V [10]. Les 3 dernières décades ont vu le remplacement de l'acier inoxydable par le titane et ses alliages (TA6V) [4]. Un nouveau bracket de titane pur grade 4 a été produit au milieu des années 1990 pour répondre à la corrosion des aciers inoxydables et à la demande croissante en Europe de matériaux alternatifs sans nickel [11]. À cause de leur résistance à la corrosion supérieure comparée aux autres alliages, à leur non carcinogénicité, hyposensibilité et excellente biocompatibilité, le titane et ses alliages peuvent être implantés de manière permanente dans la région maxillofaciale [4]. Ils tendent même à être une alternative aux alliages précieux et alliages non précieux à base de nickel et de cobalt, en prothèse conjointe conventionnelle, technique céramo-métallique et prothèse partielle amovible.

Un fil orthodontique en acier inoxydable pauvre en nickel $(10 \%)$ développé, riche en nitrogène $(0,41 \% \mathrm{~N})$ est préféré à un acier inoxydable 316L [25]. Toutefois, la durée d'un traitement orthodontique avec des matériaux sans nickel est légèrement plus longue [17]. Les couronnes pédodontiques préformées en alliage $\mathrm{NiCr}$ avec près de $72 \%$ de nickel sont aussi maintenant remplacées par des couronnes en acier inoxydable dont les concentrations en nickel sont limitées à 9-12\% [26]. 


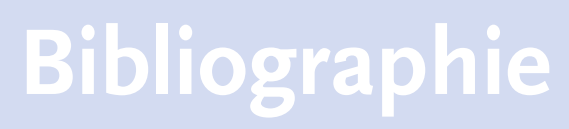

[1] Setcos JC et al. The safety of nickel containing dental alloys. Dent Mater 2006 ; 22 : 1163-1168.

[2] Eliades T. Orthodontic materials research and applications : Part 2. Current status and projected future developments in materials and biocompatibility. Am J Orthod Dentofacial Orthop $2007 ; 131: 253-262$.

[3] Schmalz G et al. Biological interactions of dental cast alloys with oral tissues. Dent Mater $2002 ; 18: 396-406$.

[4] Haug R. H, Retention of asymptomatic bone plates used for orthognathic surgery and facial fractures. J Oral Maxillofac Surg 1996; 54 (5) : 611-617.

[5] House K et al. Corrosion of orthodontic appliance - should we care ? Orthod Dentofacial Orthop 2008 ; 133 : 584-592.

[6] Vahter $\mathrm{M}$ et al. Gender differences in the disposition and toxicity of metals. Environmental Research 2007 ; 104 : 85-95.

[7] Wataha JC. Biocompatibility of dental casting alloys : a review. J Prothet Dent 2000 ; $83: 223-234$.

[8] Arikan A. Effects of nickel-chrome dental alloys used in dentistry on saliva and serum nickel levels, peripheral T-lymphocytes and some other blood parameters. J Oral rehabil $1992 ; 19$ (4) : 343-352.

[9] Ruff CA et al. The impact of various patient factors on contact allergy to nickel, cobalt, and chromate. J AmAcad Dermatol 2006 ; 55 : 32-39.
[10] Fini M, Nicoli Aldini N, Torricelli P, Giavaresi G, Borsari V, Lenger $\mathrm{H}$ et al. A new austenitic stainless steel with negligible nickel content : an in vitro and in vivo comparative investigation. Biomater $2003 ; 24: 4929-4939$.

[11] Whitley JQ et al. Resistance to sliding of titanium brackets tested against stainless steel and beta-titanium archwires with second-order angulation in the dry and wet states. Am J Orthod Dentofacial Orthop 2007 ; 131 (3) : 400411.

[12] Reclaru L et al. Comparative corrosion study of 'Ni-free' austenitic stainless steels in view of medical applications. Acta Biomater 2006 ; 2 (4) : 433-444.

[13] INRS. Ateliers de fabrication de prothèses dentaires. Guide pratique de ventilation $\mathrm{N}^{\circ} 16$. Available from : URL : http://www.inrs.fr/ media.html?refINRS=ED\%20760.

[14] AFFSAPS. Guide pour la mise sur le marché de dispositifs médicaux sur mesure appliqué au secteur dentaire. Exigences du livre II titre $1^{\text {er }}$ du code de la Santé Publique. Available from : URL : www.prodental.org/ upload/documents_fichier_230.pdf

[15] Eliasson A et al. A clinical evaluation of cobalt-chromium metal-ceramic fixed partial dentures and crowns : a three-to-seven-year retrospective study. J Prosthet Dent 2007 ; 98 : 6-16.

[16] Krantz A et al. Metal exposure and common chronic diseases : a guide for the clinician. Dis Mon 2004 ; 50 (5) : 220-262.

[17] Sakai Y et al. A case of severe crowding with nickel allergy, treated using nonnickel-containing orthodontic appliances. Orthod Waves 2009, doi : 10.1016/j. odw.2009.01.003.
[18] Grande NM et al. Cross-sectional analysis of root canals prepared with NiTi rotary instruments and stainless steel reciprocating files. Oral Surg Oral Med Oral Pathol Oral Radiol Endodont 2007 ; 103 (1) : 120-126.

[19] Mockers O et al. Cytotoxicity of orthodontic bands, brackets and archwires in vitro. Dent Mater 2002 ; 18 : 311-317.

[20] Faccioni F et al. In vivo study on metal release from fixed orthodontic appliances and DNA damage in oral mucosa cells. Am J Orthod Dentofacial Orthop 2003 ; 124 : 687-694.

[21] Virtanen $S$ et al. Special modes of corrosion under physiological and simulated physiological conditions. Acta Biomater 2008; $4: 468-476$.

[22] Yfantis C et al. Analytical and electrchemical evaluation of the in vitro corrosion behavior of nickel-chrome and cobalt-chrome casting alloys for metal-ceramic restorations. Eur J prosthodont Rest Dent 2007 ; 15 (1) : 33-40.

[23] Mareci D et al. The electrochemical behaviour of various non-precious $\mathrm{Ni}$ and $\mathrm{Co}$ based alloys in artificial Saliva. Aur Cell Mater $2005 ; 10$ (10) : 1-7.

[24] Bilhan et al. The effect of mucine, IgA, urea, and lysozyme on the corrosion behavior of various non-precious dental alloys and pure titanium in Artificial Saliva. J Biomater Appl $2007 ; 22$ : 197-221.

[25] Niimoni M. fatigue characteristics of metallic biomaterials. Int J Fatigue 2007 ; 992 1000.

[26] Zinelis S et al. Morphological and compositional alterations of in vivo aged prefabricated pediatrical metal crowns (PMCs). Dent Mater 2008 ; 24 : 216-220. 